\title{
Robust Course Keeping Control of a Fully Submerged Hydrofoil Vessel with Actuator Dynamics: A Singular Perturbation Approach
}

\author{
Sheng Liu, Changkui Xu, and Lanyong Zhang \\ College of Automation, Harbin Engineering University, Harbin 150001, China \\ Correspondence should be addressed to Changkui Xu; xuchangkui@hrbeu.edu.cn
}

Received 27 May 2017; Accepted 26 July 2017; Published 5 September 2017

Academic Editor: Shoudong Huang

Copyright (C) 2017 Sheng Liu et al. This is an open access article distributed under the Creative Commons Attribution License, which permits unrestricted use, distribution, and reproduction in any medium, provided the original work is properly cited.

\begin{abstract}
This paper presents a two-time scale control structure for the course keeping of an advanced marine surface vehicle, namely, the fully submerged hydrofoil vessel. The mathematical model of course keeping control for the fully submerged hydrofoil vessel is firstly analyzed. The dynamics of the hydrofoil servo system is considered during control design. A two-time scale model is established so that the controllers of the fast and slow subsystems can be designed separately. A robust integral of the sign of the error (RISE) feedback control is proposed for the slow varying system and a disturbance observer based state feedback control is established for the fast varying system, which guarantees the disturbance rejection performance for the two-time scale systems. Asymptotic stability is achieved for the overall closed-loop system based on Lyapunov stability theory. Simulation results show the effectiveness and robustness of the proposed methodology.
\end{abstract}

\section{Introduction}

With the development of science and technology, maritime transport has entered a high-speed era. As an advanced marine surface vehicle, the fully submerged hydrofoil vessel (FSHV) can cruise at a high speed under rough sea wave. The lift force of the hydrofoils generated by the high-speed fluid can elevate the ship hull from the water, which highly reduces the wave resistance and friction to the ship [1-3]. However, it also leads to instability of the open loop system of the FSHV. Therefore, an autopilot is necessary for this type of hydrofoil vessels. Currently, the commercial control system equipped on board is based on optimal control theory which has a weak disturbance rejection property [3-5]. For the sake of the high cruising speed, the nonlinear hydrodynamic damping of the FSHV cannot be neglected [6], so that the widely used model of marine surface vessels can be no longer applicable for the existence of strong coupling between yaw and roll dynamics. Moreover, the model uncertainties and stochastic disturbance caused by wind, waves and currents also make it difficult for precise steering of this species of marine vehicle.

In fact, the path of marine vessels is usually straight line or straight lines formed by waypoints, on which condition there is little coupling between the heave/pitch dynamics and the yaw/roll dynamics. Some literatures have been investigated about the riding control $[3,4]$. This paper mainly focuses on the steering control of the FSHV.

As for the nonlinear steering control for marine vessels, a series of control methodologies have been explored, such as advanced sliding mode control [7-9], robust control [10, 11], and adaptive control [12-14]. But these passive disturbance attenuation control methods may suffer from actuator chattering, system conservation, derivative explosion, and rigorous proof for stability. In some literatures, intelligent approximations, such as fuzzy logic [15] and neural network [16], are introduced as feedforward components for estimation and disturbance compensation. But the convergence rate of weight function and the problem of extremum solving still remain to be settled. Disturbance rejection control is widely applied in rigid-body dynamics and servo systems [17-19]. Disturbance observer based control (DOBC) approaches can estimate the generalized disturbances and improve the robustness of the systems in an active way. Control applications in many different fields have validated the effectiveness of the DOBC, such as manipulator tracking control [20], power system control $[17,18]$, and missile guidance and 
control $[19,21]$. Due to the simple structure of the DOB, it is often used in real-time control applications for high precision. To enhance the disturbance rejection performance, a composite hierarchical antidisturbance control (CHADC) is proposed in [22-25]. In this method, the disturbance observer is built in the inner-loop to estimate the generalized disturbance, and an outer-loop robust controller is also developed to compensate for the residual error of the DOB [22]. However, time scale separation and cascade analysis for multi-time scale subsystem are not included.

In the existing literatures about motion control of marine vehicles with actuator dynamics, the actuator system is always regarded as a first-order inertial element [26]. However, most actuator systems are servo systems driven by electric motors or electronic-hydraulic mediums. The first-order inertial model cannot present the main features of these servo systems since there are high-order dynamic elements and model uncertainties in the servo systems, and most servo systems do not have static stability. Therefore, the controller for the inner-loop needs to be designed additionally. Current approaches for control design together with actuator dynamics are based on backstepping and cascade system theory, which consider the rigid-body dynamics and the actuator dynamics as an integrated system [26], so that the control input can be calculated directly. However, these methodologies take little emphasis on the interconnection between actuator dynamics and attitude stabilization, as well as the closed-loop stability analysis for the overall system with different time scales. The main reasons for this problem are listed as follows.

(1) In most cases, adding the servo system for the actuator mechanism into the control objective will lead to the increase of the relative degree of the overall system, which will cause the so-called "explosion of complexity," such that backstepping based control strategies are no longer conventional.

(2) The frequency response of the actuator servo system is different from that of the attitude tracking system. Therefore, it is not advisable to make the system control design using classical state feedback control methodologies just in a simply single scheme.

(3) External disturbance and model uncertainties exist in both of the systems mentioned above, where their physical characteristics are different according to the time scales of the attitude tracking subsystem and the actuator subsystem. In order to guarantee the control precision and disturbance rejection performance, time scale separation is required to justify the implementation of the controller design for each subsystem.

When the actuator dynamics is considered in the control design for the course keeping control of the FSHV, multi-time scale phenomenon tends to be particularly sensitive for the interconnection analysis of the overall system. Singular perturbation approach is such a method to analyze and separate different time scale motions in control problems [27-30]. In this approach, by introducing a small time scale parameter $\varepsilon$, the overall system is decomposed into two different time scale subsystems, namely, the quasi-steady-state (slow) subsystem and the boundary-layer (fast) subsystem. For the course keeping control of the FSHV, the attitude stabilization for rigidbody dynamics is obviously the slow dynamic subsystem while the actuator servo system is the fast dynamic subsystem since natural frequency of the servo system is definitely much higher than the rigid-body dynamics and the response time of the servo system to a steady state is much less than the rigid-body dynamics of the FSHV for the different inertias of these subsystems. Accordingly, to analyze the characteristic of the multi-time scales and design a control strategy without the drawbacks of the aforementioned high-order controllers, a singular perturbation based hierarchical control structure is required for the compound system.

The basis of singular perturbation theory can be referred to [27]. And this methodology has been applied in nonlinear systems such as control practices in aerospace and UAV systems [27-30] for many years. However, to the best of our knowledge, although singular perturbation method has been reported for motion control of conventional marine vessels [31, 32], applications on multi-degree-of-freedom stabilization of marine vessels with actuator dynamics can rarely be seen in the open literatures. Moreover, in the literatures above, the proposed controllers for the fast and slow subsystems are basic state feedback methods and feedback linearization based approaches, which are designed based on the nominal models of the objective systems without the consideration of the lumped disturbances. Therefore, the disturbance rejection performance cannot be guaranteed. For the complex motion control of marine vehicles, the disturbances caused by winds, waves, and currents severely affect the control performance and precision, so the robustness of the closed-loop system must be particularly evaluated in control design.

In this paper, a hierarchical robust control structure is proposed for the course keeping of the FSHV with actuator dynamics against composite disturbances. The main contributions are listed as follows.

(1) Different from the CHADC methodology, singular perturbation theory is used for the time scale separation of the lateral dynamics of the FSHV and the actuator servo system to explore the interconnection of the slow dynamics and the fast dynamics.

(2) A robust integral of the sign of the error (RISE) feedback control is presented for the stabilization of the slow-time scale subsystem and a DOB based feedback control is utilized for the fast-time scale subsystem. All control signals are continuous which are practical for engineering implementation.

(3) Disturbance attenuation performance is guaranteed in both quasi-steady-state subsystem and boundarylayer subsystem. Interconnection of the subsystems is analyzed and uniformly asymptotic stability is achieved at the equilibrium point.

The rest of the paper is organized as follows. In Section 2, the mathematical models of the lateral dynamics of a FSHV 

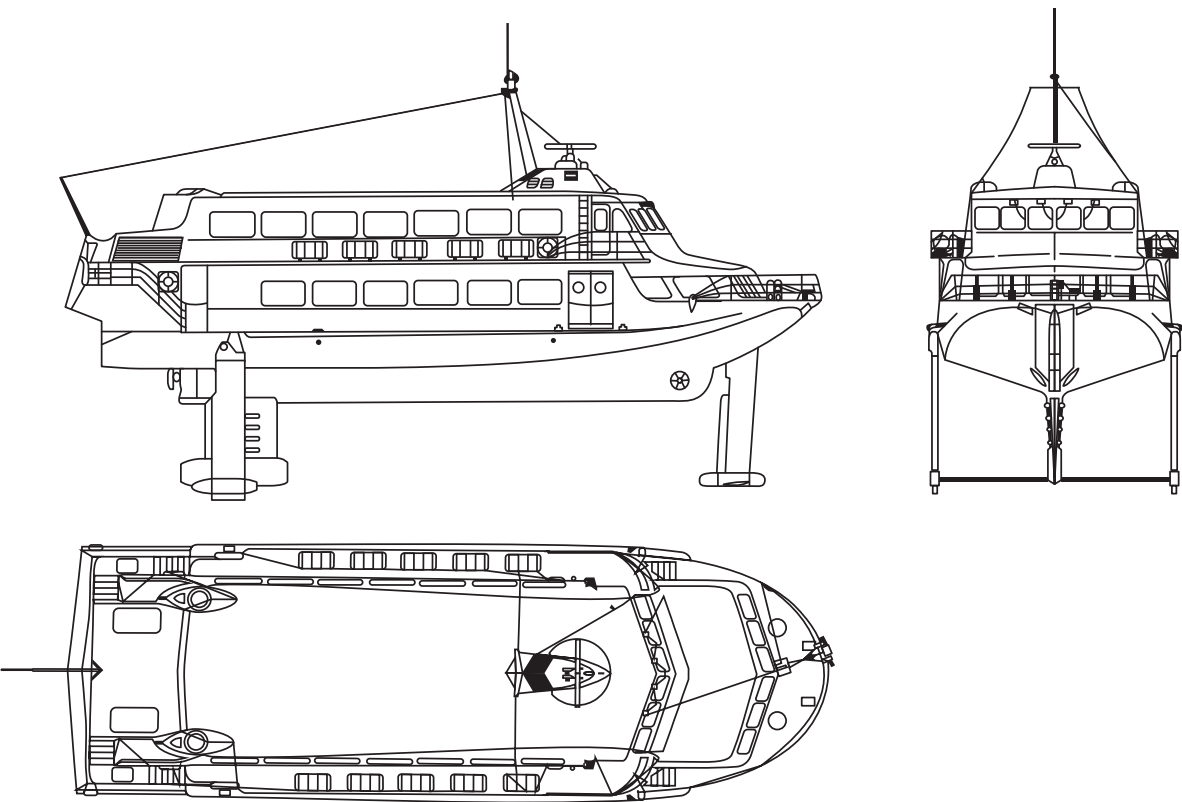

FIGURE 1: Fully submerged hydrofoil vessel.

and its hydrofoil servo system are developed. In Section 3, a hierarchical robust disturbance rejection control strategy is proposed based on singular perturbation theory. Stability of the hierarchical system is analyzed via Lyapunov stability theory. Simulation is carried out in Section 4 to validate the effectiveness of the proposed control method, followed by conclusions in Section 5.

\section{Problem Formulation}

A typical configuration of a fully submerged hydrofoil vessel is shown in Figure 1. The T-shaped bow foil is equipped with two controlled flaps, acting together. The aft foil has a pair of central flaps and two pairs of ailerons. Struts of the aft foil are equipped with rudders, which is used for roll and yaw dynamics together with the ailerons. And the bow foil and the central part of the aft foil are for longitudinal motion control.

Assuming that the surge speed is controlled by an individual propulsion system, the maneuvering model of a typical marine vehicle is shown as follows:

$$
\begin{aligned}
\dot{\eta} & =J(\eta) v, \\
M \dot{v}+C\left(u_{0}, v\right) v+D\left(u_{0}, v\right) v+G(\eta) & =B u+\tau_{d}
\end{aligned}
$$

where $\eta \in[\phi, \psi]^{T}$ is a vector of position and orientation with coordinates in the earth-fixed frame and $v \in[p, r]^{T}$ is a vector of linear and angular velocities with coordinates in the bodyfixed frame.

$$
J=\left[\begin{array}{cc}
1 & 0 \\
0 & \cos \phi
\end{array}\right]
$$

is the Jacobian transformation matrix related to the above frames. $M \in R^{2 \times 2}$ denotes the inertia including added mass.

$$
M=\left[\begin{array}{cc}
I_{x}-K_{\dot{p}} & m x_{g}-K_{\dot{r}} \\
m x_{g}-N_{\dot{p}} & I_{z}-N_{\dot{r}}
\end{array}\right] .
$$

$C\left(u_{0}, v\right)$ is the Coriolis and centripetal matrix with the following form:

$$
C\left(u_{0}, v\right)=\left[\begin{array}{cc}
0 & 0 \\
0 & m x_{g} u_{0}
\end{array}\right] .
$$

$D\left(u_{0}, v\right)$ is the coupling interactions caused by the nonlinear hydrodynamic damping, which is expressed as

$$
D\left(u_{0}, \nu\right)=\left[\begin{array}{cc}
-K_{p} & -K_{r}-K_{r \phi \phi} \phi^{2} \\
-N_{p} & -N_{r}-N_{r r \phi} r \phi
\end{array}\right],
$$

where $K_{p}, K_{r \phi \phi}$, and $K_{r}$, and $N_{p}, N_{r}$, and $N_{r r \phi}$ are the hydrodynamic coefficients. On the low-speed mode, however, the high-order terms and coupling interactions among the forces from each DOF are not considered; that is, $D\left(u_{0}, v\right)$ is often regarded as a linear term, even canceled in many literatures.

$G(\eta)$ represents the gravity term which is described as $G(\eta)=\left[W \overline{G M_{T}}, 0\right]^{T}$, where $W=m g$ is the weight and $\overline{G M_{T}}$ is the transverse metacenter height.

$$
B=\left[\begin{array}{ll}
K_{\delta_{R}} & K_{\delta_{A}} \\
N_{\delta_{R}} & N_{\delta_{A}}
\end{array}\right]
$$

is the control moment coefficient matrix subjected to the control surface, and $\delta=\left[\begin{array}{ll}\delta_{R} & \delta_{A}\end{array}\right]^{T}$ is the control input, where $\delta_{R}$ and $\delta_{A}$ represent the rudder angle and aileron angle of the hydrofoil system. 


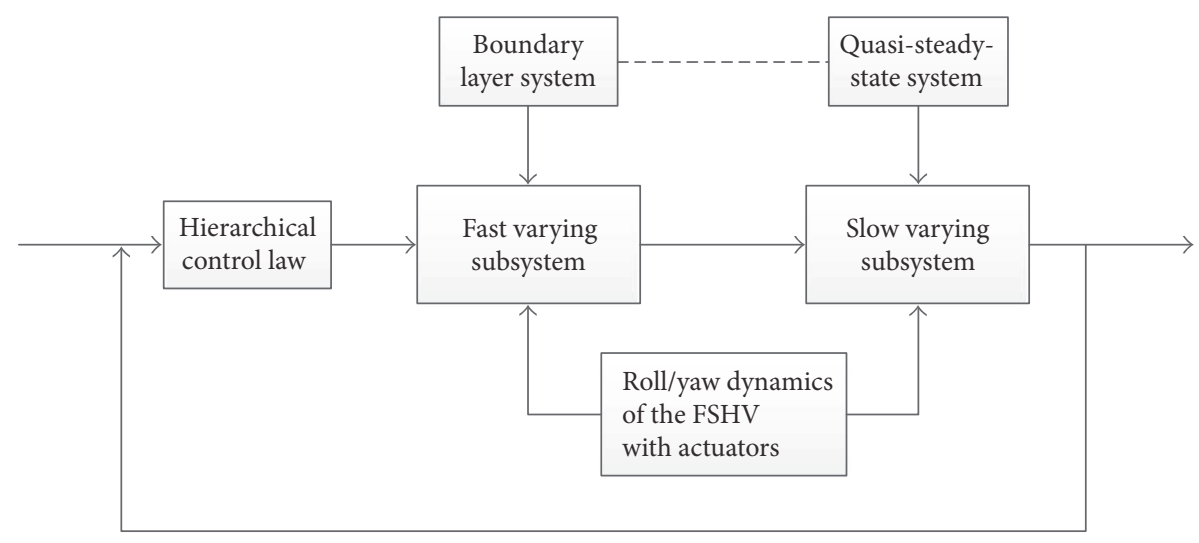

FIgURE 2: Control structure for the course keeping of FSHV.

By substituting (16) into (17), we rewrite the dynamics of the FSHV as follows:

$$
\begin{aligned}
M^{*} \ddot{\eta}+C^{*}\left(u_{0}, v, \eta\right) \dot{\eta}+D^{*}\left(u_{0}, v, \eta\right) \dot{\eta}+G^{*}(\eta) \\
\quad=J^{-T}(\eta)\left(B \delta+\tau_{d}\right),
\end{aligned}
$$

where $M^{*}=J^{-T} M J^{-1}, C^{*}=J^{-T}\left[C-M J^{-1} \dot{J}\right] J^{-1}, D^{*}=$ $J^{-T} D J^{-1}$, and $G^{*}=J^{-T} G$.

Define $\left[x_{1}, x_{2}\right]^{T} \triangleq[\eta, \dot{\eta}]^{T}$; then (18) can be rewritten into the following form:

$$
\begin{aligned}
& \dot{x}_{1}=x_{2}, \\
& \dot{x}_{2}=\bar{F}\left(x_{1}, x_{2}\right)+\bar{B}\left(x_{1}, x_{2}\right) \delta+\bar{\tau}_{d},
\end{aligned}
$$

where $\bar{F}\left(x_{1}, x_{2}\right)=-M^{*-1}\left[C^{*} x_{2}+D^{*} x_{2}+G^{*}\left(x_{1}\right)\right], \bar{B}\left(x_{1}, x_{2}\right)=$ $M^{*-1} J^{-T} B$, and $\bar{\tau}_{d}=M^{*-1} J^{-T} \tau_{d}$.

If the parameter uncertainties are considered in the modeling and control of the FSHV, the following notation is introduced:

$$
\bar{F}\left(x_{1}, x_{2}\right)=\bar{F}_{0}\left(x_{1}, x_{2}\right)+\bar{F}_{\Delta}\left(x_{1}, x_{2}\right),
$$

where subscript 0 denotes the nominal part of the corresponding matrix and the subscript $\Delta$ represents the perturbed part of the system dynamics. The yaw/roll dynamics of the FSHV with model uncertainties can be rewritten as follows:

$$
\begin{aligned}
& \dot{x}_{1}=x_{2}, \\
& \dot{x}_{2}=\bar{F}_{0}\left(x_{1}, x_{2}\right)+\bar{B}\left(x_{1}, x_{2}\right) \delta+f_{1}(t),
\end{aligned}
$$

where $f_{1}(t)=\bar{F}_{\Delta}\left(x_{1}, x_{2}\right)+\bar{\tau}_{d}$.

As to the actuator system, AC motor based electric servo solution and hydraulic servo scheme are often used for the implementation of the hydrofoil servo system $[5,6]$. Without loss of generality, a typical second-order system is established for the mathematical model of the hydrofoil transmission system as follows:

$$
\ddot{\delta}=-a_{2} \dot{\delta}+b_{2} u_{c}+d_{2}(t)
$$

Define $z_{1} \triangleq \delta \in R^{2}$ and $z_{2} \triangleq \dot{\delta} \in R^{2}$. Considering the model uncertainties of the actuator dynamics, (11) can be rewritten as the following form:

$$
\begin{aligned}
& \dot{z}_{1}=z_{2}, \\
& \dot{z}_{2}=-a_{2} z_{2}+b_{2} u_{c}+f_{2}(t),
\end{aligned}
$$

where $a_{2}=\operatorname{diag}\left[a_{21}, a_{22}\right]$ represents the speed factor of the servo system; $b_{2}=\operatorname{diag}\left[b_{21}, b_{22}\right]$ is the gain of the power amplifier for the servo system. $u_{c}=\left[u_{c 1}, u_{c 2}\right]^{T}$ is the flap command of the hydrofoil transmission system, and $f_{2}(t)=$ $d_{2}(t)-\Delta a_{2} z_{2}$ is the composite disturbance caused by model uncertainties and external wave disturbance, and $\Delta a_{2}$ is the perturbation item of $a_{2}$.

The control objective is to stabilize the maneuvering dynamics of the FSHV for the task of course keeping based on time scale separation and singular perturbation control theory. The control scheme of the overall system is summarized in Figure 2. For the subsequent control design, the following assumptions are brought in for convenience.

Assumption 1. The nonlinear functions $M(\cdot), C(\cdot), D(\cdot)$, and $G(\cdot)$ are continuously differentiable and locally Lipschitz.

Assumption 2. The generalized disturbances $f_{1}(t), f_{2}(t)$ are bounded, and $f_{2}(t)$ varies slowly.

\section{Time Scale Separation and Control Design}

3.1. Multi-Time Scale Analysis of the FSHV. In this section, the multi-time scale decomposition of the full model (10) is analyzed. Generally, in marine vehicle control problems, no singular perturbation parameter appears explicitly in the dynamical model of the marine vessels. As for the fully submerged hydrofoil vessels, the mathematical model is more complex than that of the ordinary marine vessels, making it more difficult for the modeling of the singular perturbation system. In such cases, a singular perturbation parameter may be artificially inserted to define a rapid response of a certain dynamics. In other cases, this parameter may be inserted to suppress the variables in the equations that are expected to have relatively slight effects on the original system model. 
The slow-fast time scale character is often associated with a small parameter multiplying some of the state variables of the state equations describing a physical system. However, usually that parameter may not be identifiable at all and only by physical insight and experiences does one know the details of fast and slow dynamics for the system.

Experience implies that the yaw and roll dynamics are slow relative to the dynamics of the servo system for flap mechanism among the state variables of the proposed mathematical model of the FSHV, which is the motivation to establish a singular perturbation control scheme as follows:

$$
\begin{aligned}
\dot{x}_{1} & =x_{2} \\
\dot{x}_{2} & =\bar{F}_{0}\left(x_{1}, x_{2}\right)+\bar{B}\left(x_{1}, x_{2}\right) z_{1}+f_{1}(t), \\
\varepsilon \dot{z}_{1} & =\theta z_{2} \\
\varepsilon \dot{z}_{2} & =-\bar{a}_{2} z_{2}+\bar{b}_{2} u_{c}+\bar{f}_{2}(t),
\end{aligned}
$$

where $\theta=\varepsilon, \bar{a}_{2}=\varepsilon a_{2}, \bar{b}_{2}=\varepsilon b_{2}, \bar{f}_{2}(t)=\varepsilon f_{2}(t)$, and $0<\varepsilon \ll 1$ is the artificial parameter for singularly perturbed system analysis. Hence, the overall system can be divided into a slow varying subsystem $\sum_{S}(\dot{x}=f(t, x, z, \varepsilon))$ and a fast varying subsystem $\sum_{F}(\varepsilon \dot{z}=g(t, x, z, \varepsilon))$ as follows:

$$
\begin{gathered}
\sum_{S}\left\{\begin{array}{l}
\dot{x}_{1}=x_{2} \\
\dot{x}_{2}=\bar{F}_{0}\left(x_{1}, x_{2}\right)+\bar{B}\left(x_{1}, x_{2}\right) z_{1}+f_{1}(t),
\end{array}\right. \\
\sum_{F}\left\{\begin{array}{l}
\varepsilon \dot{z}_{1}=\theta z_{2} \\
\varepsilon \dot{z}_{2}=-\bar{a}_{2} z_{2}+\bar{b}_{2} u_{c}+\bar{f}_{2}(t) .
\end{array}\right.
\end{gathered}
$$

According to the two-time scale structure of the FSHV system with actuator dynamics, a hierarchical control strategy can be deployed for the maneuvering control of the FSHV. Before the following control design, a brief introduction of the singular perturbation control theory is given for convenience.

Singular perturbations cause a multi-time scale behavior of dynamical systems characterized by the presence of slow and fast transients in the system's response to external stimuli. Loosely speaking, the slow response is appropriated by the reduced model, while the discrepancy between the response of the reduced model and the full model is the fast transient. To see this point, let us consider the following generalized state equations:

$$
\begin{aligned}
\dot{x} & =f(t, x, z, \varepsilon), \\
x\left(t_{0}\right) & =x_{0}(\varepsilon), \\
\varepsilon \dot{z} & =g(t, x, z, \varepsilon), \\
z\left(t_{0}\right) & =z_{0}(\varepsilon) .
\end{aligned}
$$

Let $\varepsilon=0$; we can get

$$
0=g(t, \bar{x}, \bar{z}, 0)
$$

where the bar is used to indicate that the variables belong to a system with $\varepsilon=0$. Then it yields the quasi-steady-state equilibrium of the fast dynamics in (17); namely,

$$
\bar{z}=h(t, \bar{x}) .
$$

Substituting (19) into (16), we can get

$$
\dot{\bar{x}}=f(t, \bar{x}, h(\bar{x}), 0),
$$

which is called quasi-steady-state subsystem.

Stretching the time to $\tau=t / \varepsilon$, the fast subsystem becomes

$$
\frac{d z}{d \tau}=g(x, z(\tau)), \quad \tau=\frac{t}{\varepsilon},
$$

which is called boundary-layer subsystem. It describes the fast dynamics in a stretched time scale, where $x$ can be regarded as a constant parameter.

Since the system is separated into the quasi-steady-state subsystem and the boundary-layer subsystem, the control strategy can be designed individually for each subsystem; namely, the hierarchical control law is integrated by two parts as follows:

$$
u_{c}=u_{s}+u_{f}
$$

where $u_{s}$ is designed for the slow varying subsystem and $u_{f}$ is for the fast varying subsystem.

3.2. Control Design for the FSHV. In this section, the composite control law is designed individually for the slow subsystem and fast subsystem. Inspired by [28], the fast control law is designed first to stabilize the fast varying system. The feasibility of this proposed procedure will be analyzed afterwards.

Because of the disturbance property of the actuator servo system, a disturbance observer based state feedback controller is designed for the fast subsystem as

$$
u_{f}=\bar{b}_{2}^{-1}\left(c_{1} z_{1}+c_{2} z_{2}-\widehat{\bar{f}}_{2}\right) \text {, }
$$

where $c_{1}, c_{2}$ are two control gain matrices with appropriate dimensions to be determined; $\widehat{\bar{f}}_{2}$ is the estimate of $\bar{f}_{2}$. And the disturbance observer is presented as follows:

$$
\begin{aligned}
\widehat{\bar{f}}_{2} & =\xi+l z_{2}, \\
\dot{\xi} & =-l \xi-l\left(l z_{2}+\bar{a}_{2} z_{2}+\bar{b}_{2} u_{f}\right),
\end{aligned}
$$

where $\xi \in R^{2}$ is the internal state of the observer, and $l$ is the gain matrix of the observer with appropriate dimension to be determined.

Define $\tilde{f}_{2}$ as the disturbance estimate error. Differentiate $\tilde{f}_{2}$ with respect to time. Based on the assumptions above, it yields that

$$
\begin{aligned}
\dot{\tilde{f}}_{2} & =\dot{\bar{f}}_{2}-\dot{\bar{f}}_{2}=\dot{\xi}+l \dot{z}_{2} \\
& =-l\left(z_{2}+\bar{a}_{2} z_{2}+\bar{b}_{2} u_{f}\right)+l \bar{a}_{2} z_{2}+l \bar{b}_{2} u_{f}+l \bar{f}_{2} \\
& =-l \hat{\bar{f}}_{2}+l \bar{f}_{2}=-l \dot{\tilde{f}}_{2},
\end{aligned}
$$


which implies that the disturbance estimate error can converge to zero asymptotically if the gain matrix is Hurwitz.

Remark 3. Sometimes the external disturbance is fast varying; namely, the derivative of the disturbance is not zero. In this condition, the error dynamics of disturbance estimation still can be ultimately uniformly bounded [20, 21, 25]. The bound of the estimate error can be arbitrarily small by approximately selecting the gain function.

Despite the fast control law (23) being established first, the fast varying subsystem together with the above control law remains to be a standard singular perturbation system, and in fact the fast controller $u_{f}$ is inactive for the quasi-steadystate subsystem $z=h\left(t, x, u_{s}\right)$ [27]. Analysis for this point is derived as follows.

Rewriting subsystem $\sum_{F}$ in a block form by substituting (23) into (15) results in

$$
\varepsilon\left[\begin{array}{l}
\dot{z}_{1} \\
\dot{z}_{2}
\end{array}\right]=\left[\begin{array}{cc}
0 & \theta \\
c_{1} & c_{2}-\bar{a}_{2}
\end{array}\right]\left[\begin{array}{c}
z_{1} \\
z_{2}
\end{array}\right]+\left[\begin{array}{c}
0 \\
\bar{b}_{2}
\end{array}\right] u_{s} .
$$

Let $\varepsilon=0$; then we can get $z=h(t, x)=\left[c_{1}^{-1} u_{s}, 0\right]^{T}$ as

$$
h(t, x)=-\bar{A}^{-1} \bar{B} u_{s}
$$

where

$$
\begin{aligned}
& \bar{A}=\left[\begin{array}{cc}
0 & \theta \\
c_{1} & c_{2}-\bar{a}_{2}
\end{array}\right], \\
& \bar{B}=\left[\begin{array}{c}
0 \\
\bar{b}_{2}
\end{array}\right] .
\end{aligned}
$$

Hence, the equation $g\left(t, x, z, c_{1} z_{1}+c_{2} z_{2}-\bar{b}_{2}^{-1} \overline{\bar{f}}_{2}+u_{s}\right)=0$ has a unique root, which satisfies the requirements for a singularly perturbed system. It is more convenient to work in the $(x, y)$ coordinate where

$$
y=z-h(t, x)
$$

because this change of variables shifts the equilibrium of the boundary-layer model to the origin. In the new coordinate, the singularly perturbed system is given by

$$
\begin{aligned}
\dot{x} & =f(x, y+h(t, x)), \\
\varepsilon \dot{y} & =g(x, y+h(t, x))-\varepsilon \frac{\partial h}{\partial x} f(x, y+h(t, x)) .
\end{aligned}
$$

The reduced system $\dot{x}=f(x, y+h(t, x))$ has equilibrium point at $x=0$ and the boundary-layer system

$$
\frac{d y}{d \tau}=g(x, y+h(t, x))=\bar{A}(y+h(t, x))+\bar{B} u_{s} .
$$

By substituting (27) into (31), it yields

$$
\frac{d y}{d \tau}=\bar{A} y
$$

Define the following Lyapunov function candidate:

$$
W=\frac{1}{2} y^{T} P y
$$

where $P$ is positively defined symmetric matrices with appropriate dimension to be determined. Then it yields

$$
\frac{\partial W}{\partial y} g(x, y+h(t, x))=\frac{1}{2} y^{T}\left(A^{T} P+P A\right) y .
$$

If there exists positively defined symmetric matrices $Q$ satisfied with $A^{T} P+P A=-Q$, then we can get

$$
\frac{\partial W}{\partial y} g(x, y+h(t, x))=-\frac{1}{2} y^{T} Q y \leq-\lambda_{\min }(Q)\|y\|^{2},
$$

where $\lambda_{\min }(Q)$ is the minimum eigenvalue of $Q$.

Hence the closed-loop boundary-layer system has an asymptotically stable equilibrium point.

Then consider control design of the slow varying subsystem. Assume $\varepsilon=0$. Then the reduced system $\dot{x}=$ $f(t, x, h(t, x), 0)$ is given by

$$
\begin{aligned}
& \dot{x}_{1}=x_{2}, \\
& \dot{x}_{2}=\bar{F}_{0}\left(x_{1}, x_{2}\right)+\bar{B}\left(x_{1}, x_{2}\right) c_{1}^{-1} u_{s}+f_{1}(t) .
\end{aligned}
$$

To stabilize the slow varying system, an adaptive RISE feedback control strategy is proposed.

Define a stabilizing error $e_{1} \in R^{2}$ as

$$
e_{1} \triangleq x_{d}-x_{1}
$$

where $x_{d}=\left[\phi_{d}, \psi_{d}\right]^{T}$

Two auxiliary tracking errors $e_{2}, r \in R^{2}$ are defined as

$$
\begin{gathered}
e_{2} \triangleq \dot{e}_{1}+\alpha_{1} e_{1}, \\
r \triangleq \dot{e}_{2}+\alpha_{2} e_{2} .
\end{gathered}
$$

Substituting (37) and (38) into (39), then it yields

$$
\begin{aligned}
r= & \ddot{x}_{d}-\bar{F}_{0}\left(x_{1}, x_{2}\right)-\bar{B}\left(x_{1}, x_{2}\right) c_{1}^{-1} u_{s}-f(t)+\alpha_{1} \dot{e}_{1} \\
& +\alpha_{2} e_{2}
\end{aligned}
$$

Define an auxiliary function $S \in R^{2}$ as

$$
S \triangleq \bar{F}_{0}\left(x_{d}, t\right)-\bar{F}_{0}\left(x_{1}, x_{2}\right)+\alpha_{1} \dot{e}_{1}+\alpha_{2} e_{2} .
$$

Then (40) can be rewritten as

$$
r=\ddot{x}_{d}+S-\bar{F}_{0}\left(x_{d}, t\right)-f(t)-\bar{B}\left(x_{1}, x_{2}\right) c_{1}^{-1} u_{s} .
$$

A RISE based controller can be designed as

$$
\begin{aligned}
& u_{s} \\
& \quad=c_{1} \bar{B}^{-1}\left(x_{1}, x_{2}\right)\left[\left(k_{1}+1\right) e_{2}-\left(k_{1}+1\right) e_{2}(0)+u_{\text {int }}\right] \\
& \dot{u}_{\text {int }}=\left(k_{1}+1\right) \alpha_{2} e_{2}+\beta \operatorname{sgn}\left(e_{2}\right),
\end{aligned}
$$


where $\beta \in R^{+}$is a constant control gain and $k_{1} \in R^{+}$is a timevarying control gain. An adaptive law is applied as follows for the time-varying gain design:

$$
\dot{k}_{1}=\alpha_{3} e_{2}^{T} r .
$$

By substituting (46) into (42), the time derivative of $r$ can be expressed as

$$
\begin{aligned}
\dot{r}= & \dddot{x}_{d}+\dot{S}-\dot{\bar{F}}_{0}\left(x_{d}, t\right)-\dot{f}(t)-\left(k_{1}+1\right) r-\dot{k}_{1} e_{2} \\
& -\beta \operatorname{sgn}\left(e_{2}\right) .
\end{aligned}
$$

Define auxiliary variables as follows:

$$
\begin{aligned}
\widetilde{N} & =\dot{S}+e_{2} . \\
N_{d} & =\dddot{x}_{d}-\dot{\bar{F}}_{0}\left(x_{d}, t\right)-\dot{f}(t),
\end{aligned}
$$

Then it yields

$$
\dot{r}=\widetilde{N}+N_{d}-e_{2}-\left(k_{1}+1\right) r-\dot{k}_{1} e_{2}-\beta \operatorname{sgn}\left(e_{2}\right) .
$$

Using (40) and (41) together with the mean value theorem, we can obtain

$$
\|\widetilde{N}\| \leq \rho(\|z\|)\|z\|,
$$

where $z \in R^{3 \times 2}$ is defined as

$$
z \triangleq\left[e_{1}^{T}, e_{2}^{T}, r^{T}\right]^{T},
$$

and $\rho(\cdot)$ is a positively defined, globally invertible, nondecreasing function.

Assuming that the generalized disturbances and the guidance command input are sufficiently smooth, the following inequalities can be obtained:

$$
\begin{gathered}
\left\|N_{d}\right\| \leq \zeta_{1}, \\
\left\|\dot{N}_{d}\right\| \leq \zeta_{2},
\end{gathered}
$$

where $\zeta_{1}, \zeta_{2} \in R^{+}$are known constants.

Define $Y(z, P) \in R^{3 \times 2+1}$ as

$$
Y \triangleq\left[z^{T}, \sqrt{P}\right]^{T},
$$

where $P\left(e_{2}, t\right) \in R$ is defined as the Filippov solution to the following differential equation:

$$
\begin{gathered}
\dot{P}=-r^{T}\left(N_{d}-\beta \operatorname{sgn}\left(e_{2}\right)\right), \\
P\left(e_{2}\left(t_{0}\right), t_{0}\right)=\beta \sum_{i=1}^{n}\left|e_{2 i}\left(t_{0}\right)\right|-e_{2}\left(t_{0}\right)^{T} N_{d}\left(t_{0}\right),
\end{gathered}
$$

where the subscript $i=1, \ldots, n$ denotes the $i$ th element of the vector. Accordingly, $P$ is expressed as

$$
P(t)=\beta \sum_{i=1}^{n}\left|e_{2 i}(0)\right|-e_{2}(0)^{T} N_{d}(0)-\int_{0}^{t} L(\sigma) d \sigma,
$$

where the auxiliary function $L(t) \in R$ is defined as

$$
L(t) \triangleq r^{T}\left(N_{d}-\beta \operatorname{sgn}\left(e_{2}\right)\right) .
$$

If the control gain $\beta$ satisfies

$$
\beta>\zeta_{1}+\frac{1}{\alpha_{2}} \zeta_{2},
$$

the following inequality can be obtained:

$$
\int_{0}^{t} L(\sigma) d \sigma L(t) \leq \beta \sum_{i=1}^{n}\left|e_{2 i}(0)\right|-e_{2}(0)^{T} N_{d}(0),
$$

which can conclude that $P(t) \geq 0$.

Theorem 4. Given the slow varying dynamics of a FSHV presented in (36), if the following conditions are satisfied:

$$
\begin{aligned}
& \alpha_{1}>\frac{1}{2}, \\
& \alpha_{2}>\frac{1}{2}, \\
& \beta>\zeta_{1}+\frac{1}{\alpha_{2}} \zeta_{2},
\end{aligned}
$$

the controller designed in (43) and (44) guarantees that all the signals of the error dynamics of the slow varying subsystem are bounded under closed-loop operation and the stabilizing error converges in the sense that

$$
\left\|e_{1}(t)\right\| \longrightarrow 0 \quad \text { as } t \longrightarrow \infty .
$$

Proof. Define the following Lyapunov function as

$$
V=\frac{1}{2} e_{1}^{T} e_{1}+\frac{1}{2} e_{2}^{T} e_{2}+\frac{1}{2} r^{T} r+P,
$$

which satisfies the following inequalities:

$$
U_{1}(Y) \leq V(Y, t) \leq U_{2}(Y),
$$

where $U_{1}(Y), U_{2}(Y) \in R$ are positively defined functions defined as

$$
\begin{aligned}
& U_{1}(y) \triangleq m_{1}\|Y\|^{2}, \\
& U_{2}(y) \triangleq m_{2}\|Y\|^{2} .
\end{aligned}
$$

Differentiate $V$ with respect to time; then it yields

$$
\dot{V}=e_{1}^{T} \dot{e}_{1}+e_{2}^{T} \dot{e}_{2}+r^{T} \dot{r}+\dot{P} .
$$

According to (37)-(48), $\dot{V}$ can be further expanded as

$$
\begin{aligned}
\dot{V}= & r^{T} \widetilde{N}-\left(k_{1}+1\right)\|r\|^{2}-\dot{k}_{1} r^{T} e_{2}-\alpha_{1}\left\|e_{1}\right\|^{2} \\
& -\alpha_{2}\left\|e_{2}\right\|^{2}+e_{1}^{T} e_{2} .
\end{aligned}
$$


Substituting the adaptive law in (47) into (64) together with (49), we can obtain

$$
\begin{aligned}
\dot{V} \leq & \rho(\|z\|)\|r\|\|z\|-\left(k_{1}+1\right)\|r\|^{2}-\alpha_{3}\left\|r^{T} e_{2}\right\|^{2} \\
& -\alpha_{1}\left\|e_{1}\right\|^{2}-\alpha_{2}\left\|e_{2}\right\|^{2}+e_{1}^{T} e_{2} .
\end{aligned}
$$

Since $2 e_{1}^{T} e_{2} \leq\left\|e_{1}\right\|^{2}+\left\|e_{2}\right\|^{2},(65)$ becomes

$$
\begin{aligned}
& \dot{V} \leq \rho(\|z\|)\|r\|\|z\|-\left(k_{1}+1\right)\|r\|^{2}-\alpha_{3}\|r\|^{2}\left\|e_{2}\right\|^{2} \\
& -\left(\alpha_{1}-\frac{1}{2}\right)\left\|e_{1}\right\|^{2}-\left(\alpha_{2}-\frac{1}{2}\right)\left\|e_{2}\right\|^{2} \\
& =-\|r\|^{2}-\left(\alpha_{1}-\frac{1}{2}\right)\left\|e_{1}\right\|^{2}-\left(\alpha_{2}-\frac{1}{2}\right)\left\|e_{2}\right\|^{2} \\
& -\alpha_{3}\|r\|^{2}\left\|e_{2}\right\|^{2} \\
& +\frac{4 k_{1}\left(-k_{1}\|r\|^{2}+\rho(\|z\|)\|r\|\|z\|\right)}{4 k_{1}} \\
& =-\|r\|^{2}-\left(\alpha_{1}-\frac{1}{2}\right)\left\|e_{1}\right\|^{2}-\left(\alpha_{2}-\frac{1}{2}\right)\left\|e_{2}\right\|^{2} \\
& -\alpha_{3}\|r\|^{2}\left\|e_{2}\right\|^{2} \\
& +\frac{-4 k_{1}^{2}\|r\|^{2}+2 \cdot 2 k_{1}\|r\| \rho(\|z\|)\|z\|}{4 k_{1}}, \\
& \dot{V} \leq-\|r\|^{2}-\left(\alpha_{1}-\frac{1}{2}\right)\left\|e_{1}\right\|^{2}-\left(\alpha_{2}-\frac{1}{2}\right)\left\|e_{2}\right\|^{2} \\
& -\alpha_{3}\|r\|^{2}\left\|e_{2}\right\|^{2} \\
& +\frac{-4 k_{1}^{2}\|r\|^{2}+4 k_{1}^{2}\|r\|^{2}+\rho^{2}(\|z\|)\|z\|^{2}}{4 k_{1}} \\
& \leq-\|r\|^{2}-\left(\alpha_{1}-\frac{1}{2}\right)\left\|e_{1}\right\|^{2}-\left(\alpha_{2}-\frac{1}{2}\right)\left\|e_{2}\right\|^{2} \\
& +\frac{\rho^{2}(\|z\|)\|z\|^{2}}{4 k_{1}} \leq-\lambda\|z\|^{2}+\frac{\rho^{2}(\|z\|)\|z\|^{2}}{4 k_{1}},
\end{aligned}
$$

where $\lambda=\min \left\{\alpha_{1}-1 / 2, \alpha_{2}-1 / 2,1\right\}$, and the bounding function $\rho(\|z\|)$ is a positive, globally invertible, nondecreasing function. Therefore, $\alpha_{1}$ and $\alpha_{2}$ must be selected based on the sufficient conditions in Theorem 4.

The expression in (67) can be further upper bounded by a continuous, positive semidefinite function

$$
\dot{V} \leq-c\|z\|^{2}=U(Y),
$$

where $c \in R$ is a positive constant.

According to the inequalities in (61) and (67), we can prove that $V(Y, t) \in L_{\infty}$. Thus $e_{1}, e_{2}, r \in L_{\infty}$. The closedloop error system can be utilized to guarantee that the remaining signals are bounded and the definition of $U(y)$ and $z(t)$ can conclude that $U(Y)$ is uniformly continuous. According to Theorem 8.4 of [24], $c\|z\|^{2} \rightarrow 0$ as $t \rightarrow \infty$. Then based on the definition of $z(t)$, it can be concluded that $\left\|e_{1}(t)\right\| \rightarrow 0$ as $t \rightarrow \infty$. Hence the asymptotical stability is achieved for the equilibrium point of the slow varying subsystem.

Theorem 5. Consider the multi-time scale mathematical model for course keeping of a FSHV with actuator dynamics in (14), (15). With a hierarchical control strategy designed in (22), (23), (24), and (43), there exists $\varepsilon^{*}>0$ so that, for all $\varepsilon<\varepsilon^{*}$, the overall system can achieve asymptotic stability at the equilibrium point.

Proof. In order to analyze the closed-loop stability of the overall system, we need to explore the interconnection between the slow varying subsystem and the fast varying subsystem.

Define a composite Lyapunov function candidate as follows:

$$
v(x, y)=(1-d) V(x)+d W(x, y), \quad 0<d<1,
$$

where the constant $d$ is to be chosen. Calculating the derivative of $v$ along the trajectories of the full system (14), (15), we can obtain

$$
\begin{aligned}
\dot{v}= & (1-d) \frac{\partial V}{\partial x} f(x, y+h(x)) \\
& +\frac{d}{\varepsilon} \frac{\partial W}{\partial y} g(x, y+h(x)) \\
& -d \frac{\partial W}{\partial y} \frac{\partial h}{\partial x} f(x, y+h(x)) \\
& +d \frac{\partial W}{\partial x} f(x, y+h(x)) \\
= & (1-d) \frac{\partial V}{\partial x} f(x, y+h(x)) \\
& +\frac{d}{\varepsilon} \frac{\partial W}{\partial y} g(x, y+h(x)) \\
& +(1-d) \frac{\partial V}{\partial x}[f(x, y+h(x))-f(x, h(x))] \\
& +d\left[\frac{\partial W}{\partial x}-\frac{\partial W}{\partial y} \frac{\partial h}{\partial x}\right] f(x, y+h(x)) .
\end{aligned}
$$

According to the former analysis,

$$
\begin{aligned}
& \frac{\partial V}{\partial x} f(x, y+h(x)) \leq-c\|z\|^{2}, \\
& \quad \text { namely } \omega_{1}=c, \psi_{1}(x)=\|z\| . \\
& \frac{\partial W}{\partial y} g(x, y+h(x)) \leq-\lambda_{\min }(Q)\|y\|^{2}, \\
& \text { namely } \omega_{2}=-\lambda_{\min }(Q), \psi_{2}(y)=\|y\| .
\end{aligned}
$$


Then the restrictions of the last two terms are derived as follows:

$$
\begin{aligned}
& \frac{\partial V}{\partial x}[f(x, y+h(x))-f(x, h(x))] \\
&= x_{1} \dot{x}_{1}+x_{2} \dot{x}_{2}-x_{1} \dot{x}_{1} \\
&+x_{2}\left(\bar{F}_{0}\left(x_{1}, x_{2}\right)+\bar{B}\left(x_{1}, x_{2}\right) c_{1}^{-1} u_{s}\right) \\
&= x_{2} \bar{B}\left(z_{1}-c_{1}^{-1} u_{s}\right) \leq \beta_{1}\|z\|\|y\| \\
& {\left[\frac{\partial W}{\partial x}-\frac{\partial W}{\partial y} \frac{\partial h}{\partial x}\right] f(x, y+h(x)) } \\
&=-c_{1}^{-1} \frac{\partial u_{s}}{\partial x_{1}} y_{1} x_{2} \\
& \quad-c_{1}^{-1} \frac{\partial u_{s}}{\partial x_{2}} y_{1}\left(\bar{F}_{0}\left(x_{1}, x_{2}\right)+\bar{B}\left(x_{1}, x_{2}\right) c_{1}^{-1} u_{s}\right) \\
& \leq-2 c_{1}^{-1} \sqrt{\frac{\partial u_{s}}{\partial x_{1}} y_{1} x_{2} \frac{\partial u_{s}}{\partial x_{2}} y_{1} \dot{x}_{2}} \\
& \leq \beta_{2}\|y\|\|z\|+\gamma\|y\|^{2} .
\end{aligned}
$$

Therefore, according to Theorem 11.3 in [24], it yields

$$
\dot{v} \leq \psi^{T}(x, y) \Lambda \psi(x, y),
$$

where $\psi(x, y)=\left[\psi_{1}(x), \psi_{2}(y)\right]^{T}$, and

$$
=\left[\begin{array}{cc}
(1-d) \omega_{1} & -\frac{1}{2}(1-d) \beta_{1}-\frac{1}{2} d \beta_{2} \\
-\frac{1}{2}(1-d) \beta_{1}-\frac{1}{2} d \beta_{2} & d\left(\left(\frac{\omega_{2}}{\varepsilon}\right)-\gamma\right)
\end{array}\right] .
$$

The right-hand side of the last inequality is a quadratic form in $\psi(x, y)$. The quadratic form is negative definite when

$$
d(1-d) \omega_{1}\left(\frac{\omega_{2}}{\varepsilon}-\gamma\right)>\frac{1}{4}\left[(1-d) \beta_{1}+d \beta_{2}\right]^{2}
$$

which is equivalent to

$$
\varepsilon<\frac{\omega_{1} \omega_{2}}{\omega_{1} \gamma+(1 / 4 d(1-d))\left[(1-d) \beta_{1}+d \beta_{2}\right]^{2}} \triangleq \varepsilon_{d} .
$$

It can be seen that the maximum value of $\varepsilon_{d}$ occurs at $d^{*}=$ $\beta_{1} /\left(\beta_{1}+\beta_{2}\right)$, which is given by

$$
\varepsilon^{*}=\frac{\omega_{1} \omega_{2}}{\left(\omega_{1} \gamma+\beta_{1} \beta_{2}\right)} .
$$

Then the origin of (14) and (15) is asymptotically stable for all $\varepsilon<\varepsilon^{*}$

\section{Simulation}

In this section, a mathematical model of a fully submerged hydrofoil vessel is applied to validate the performance of
TABLE 1: Model parameters of the FSHV.

\begin{tabular}{lcc}
\hline Parameter & Value & SI-unit \\
\hline$u_{0}$ & 23.15 & $\mathrm{~m} / \mathrm{s}$ \\
$m$ & $2.62 * 10^{5}$ & $\begin{array}{c}\mathrm{kg} \\
\mathrm{kgm}^{2}\end{array}$ \\
$I_{x}$ & $2.59 * 10^{5}$ & $\mathrm{kgm}^{2}$ \\
$I_{z}$ & $1.47 * 10^{7}$ & $\mathrm{~m}$ \\
$\overline{G M_{T}}$ & 0.025 & $\mathrm{kgm}^{2} / \mathrm{s}$ \\
$K_{p}$ & $-13.0354 * 10^{5}$ & $\mathrm{kgm}^{2} / \mathrm{s}$ \\
$K_{r}$ & $-2.4864 * 10^{5}$ & $\mathrm{kgm}^{2} / \mathrm{s}$ \\
$K_{r \phi \phi}$ & $12.5615 * 10^{5}$ & $\mathrm{kgm}^{2} / \mathrm{s}$ \\
$N_{p}$ & $-0.2182 * 10^{7}$ & $\mathrm{kgm}^{2} / \mathrm{s}$ \\
$N_{r}$ & $-1.3818 * 10^{7}$ & $\mathrm{kgm}^{2} / \mathrm{s}$ \\
$N_{r r \phi}$ & $0.9261 * 10^{7}$ & $\mathrm{kgm}^{2} / \mathrm{s}$ \\
$a_{21}$ & -15.27 & $\mathrm{kgm}^{2} / \mathrm{s}$ \\
$a_{22}$ & -38.27 & $\mathrm{kgm}^{2} / \mathrm{s}$ \\
$b_{21}$ & 22.18 & $\mathrm{kgm}^{2} / \mathrm{s}$ \\
$b_{22}$ & 32.45 &
\end{tabular}

the proposed control laws (22), (23), (24), and (43) through simulations $[1,2,5]$. The parameters of the model are given in Table 1.

In the simulation, the irregular wave disturbance is simulated based on trip theory and equivalent energy division method, with the significant wave height $H_{1 / 3}=1.5 \mathrm{~m}$. To validate the disturbance attenuation performance, a standard state feedback singular perturbation approach $[28,30]$ is established for comparison.

The command course angle is set to be $-30^{\circ}$ and $60^{\circ}$, respectively, to verify the proposed control design. The control parameters are selected as follows:

$$
\begin{aligned}
c_{1} & =\operatorname{diag}[6.75,5.41], \\
c_{2} & =\operatorname{diag}[7.56,4.89], \\
d & =0.9147, \\
l & =\operatorname{diag}[1352,835], \\
\alpha_{3} & =15.45, \\
\alpha_{1} & =40, \\
\alpha_{2} & =28, \\
\beta & =12.81, \\
\beta_{1} & =1, \\
\beta_{2} & =1, \\
\varepsilon & =0.0214 .
\end{aligned}
$$

Figures 3-6 show the roll/yaw angles and angle velocities of the FSHV with command course of -30 degrees and 60 degrees. It can be seen that during the changes of course angle, the coupling roll movement will appear, and the composite control law could stabilize this dynamics quickly and there is no reciprocating roll movement as ordinary vessel does. 


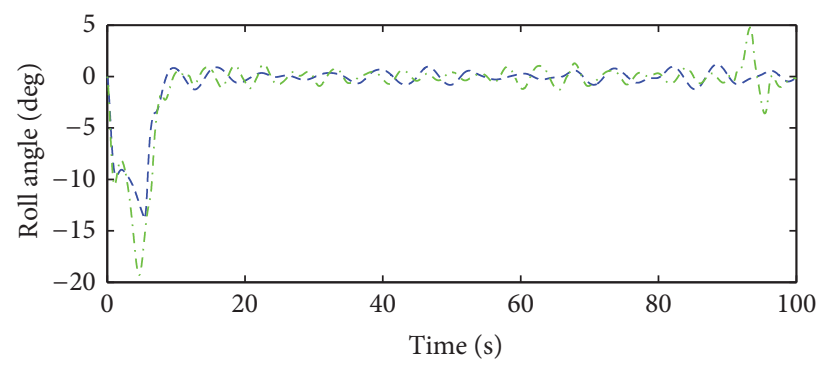

- - - Proposed control

-.. Standard feedback control

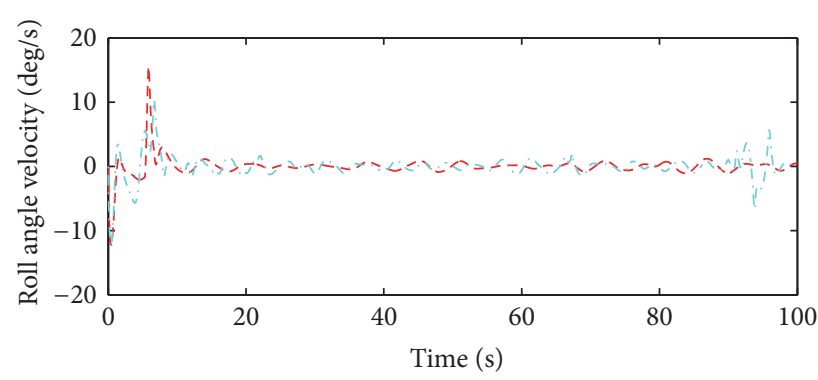

- - - Proposed control

...- Standard feedback control

FIGURE 3: Roll dynamics of the FSHV with command course angle of $-30^{\circ}$.
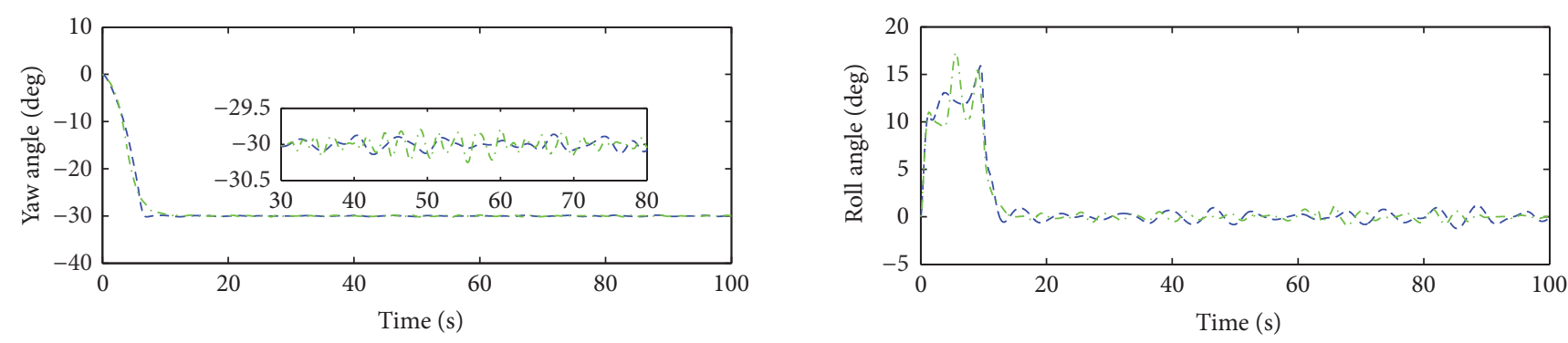

- - - Proposed control

-.- Standard feedback control

- - - Proposed control

. - . Standard feedback control
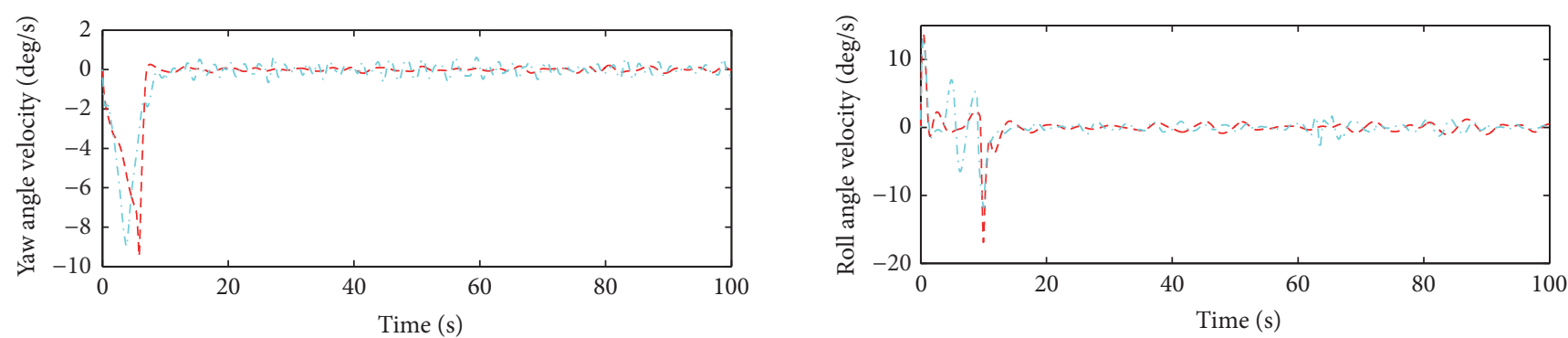

- - - Proposed control

Standard feedback control

FIgURE 4: Yaw dynamics of the FSHV with command course angle of $-30^{\circ}$.

- - - Proposed control

_. - Standard feedback control

FIGURE 5: Roll dynamics of the FSHV with command course angle of $60^{\circ}$.

According to Figures 4 and 6, with the stochastic wave disturbances, the proposed methodology acquires a faster convergence than the standard singular perturbation control. During the transient process, the roll motion using standard

singular perturbation control is much larger than that of the proposed control, and the severely reciprocating motion is more obvious, which has a negative effect on the sea-keeping capacity of the FSHV. 

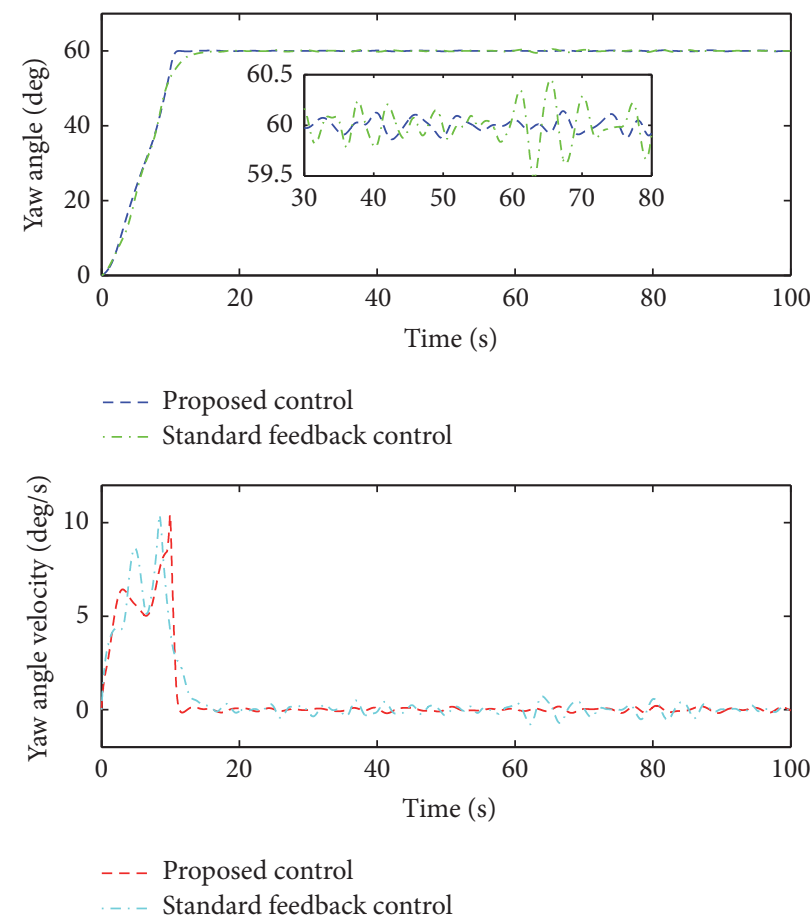

Figure 6: Yaw dynamics of the FSHV with command course angle of $60^{\circ}$.

At the steady state of the lateral dynamics, the proposed control acquires higher precision and better disturbance attenuation performance since the model uncertainties and external disturbances are considered in both slow dynamics and fast dynamics of the FSHV. The composite control law guarantees the robustness against the lumped disturbances while the control design of the standard singular perturbation approach does not take the disturbance effects into account.

From Figures 7-10, the slow-time scale and fast-time scale response of each subsystem can be seen to verify the different system characteristic of the servo system and the lateral dynamics of the FSHV. The position of the heading of arrows point out the time of convergence. When course command is generated, the servo system has a fast response to step into the steady state first. Due to the large inertia, the trajectory of the lateral dynamics of the FSHV moves relatively slower than the hydrofoil servo system.

Figures 11 and 12 show the composite control input $u_{c}$ for the system. We can see that the proposed control input is continuous, and the amplitude is lower than the standard singular perturbation approach. According to the fast dynamics in Figures 7-10 and the composite control input in Figures 11 and 12, it implies that, based on the proposed method, the robust stabilization can be guaranteed with less energy consumption. And the conservation is less than standard singular perturbation approach with no disturbance rejection performance.

\section{Conclusion}

In this paper, a two-time scale robust control structure is proposed for the course keeping control of the FSHV with
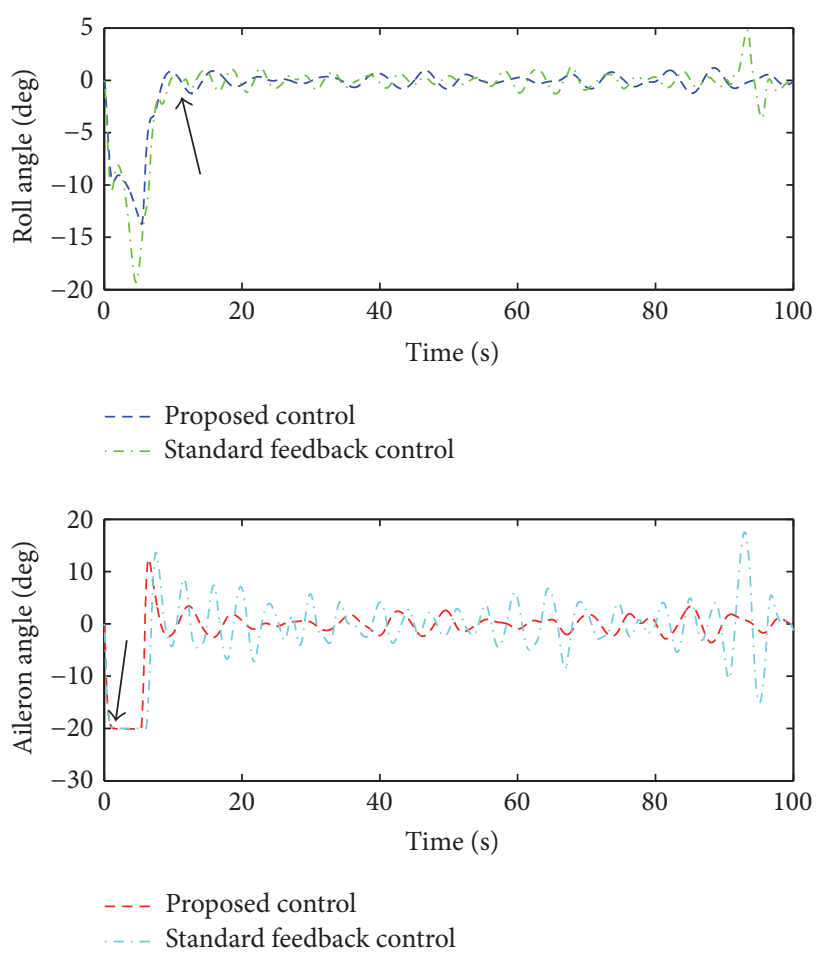

FIGURE 7: Roll response of different time scale with command course angle of $-30^{\circ}$.

actuator dynamics. We first analyze the lateral model of the FSHV as well as the hydrofoil servo system. Then a two-time scale separated model is established for the hierarchical control design. A RISE feedback control is designed for the slow 

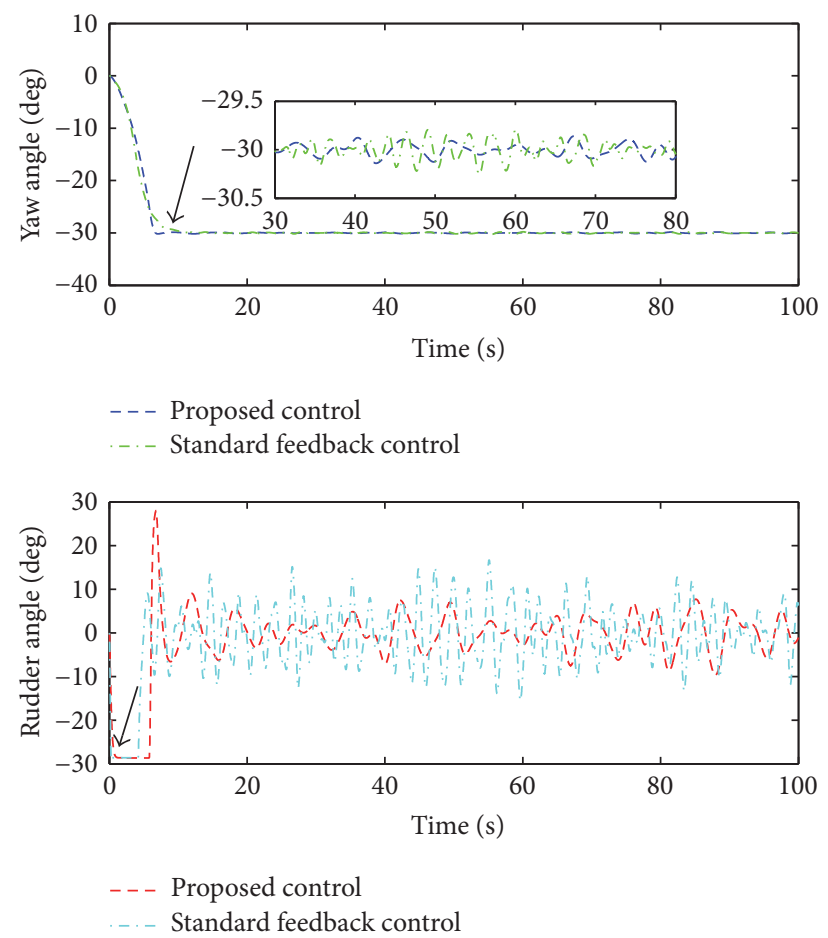

FIgURE 8: Yaw response of different time scale with command course angle of $-30^{\circ}$.

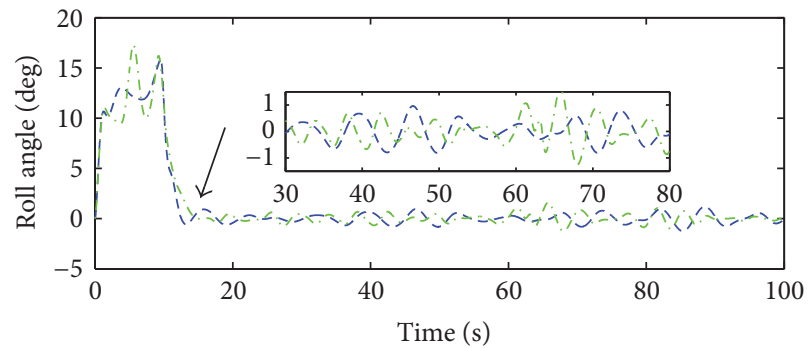

- - - Proposed control

-. - Standard feedback control

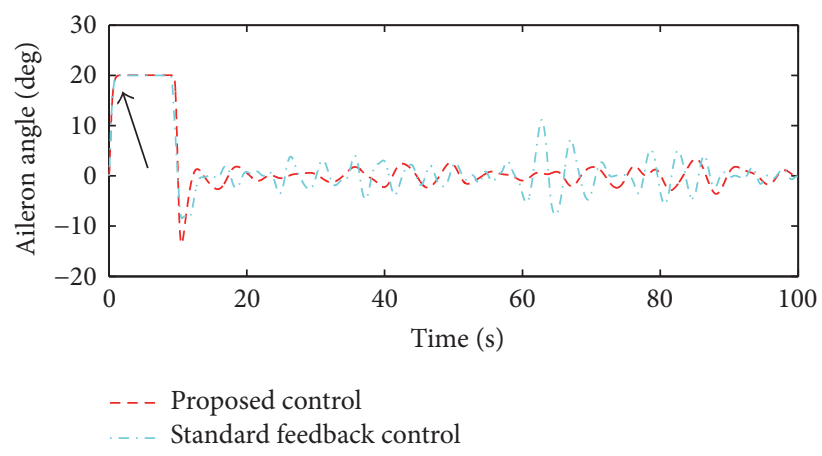

FIGURE 9: Roll response of different time scale with command course angle of $60^{\circ}$.

varying subsystem and a DOB based state feedback control is used for the fast varying subsystem to achieve disturbance attenuation performance. Uniformly asymptotic convergence is achieved for the overall system. Simulation results indicate the effectiveness of the time separation method, which shows
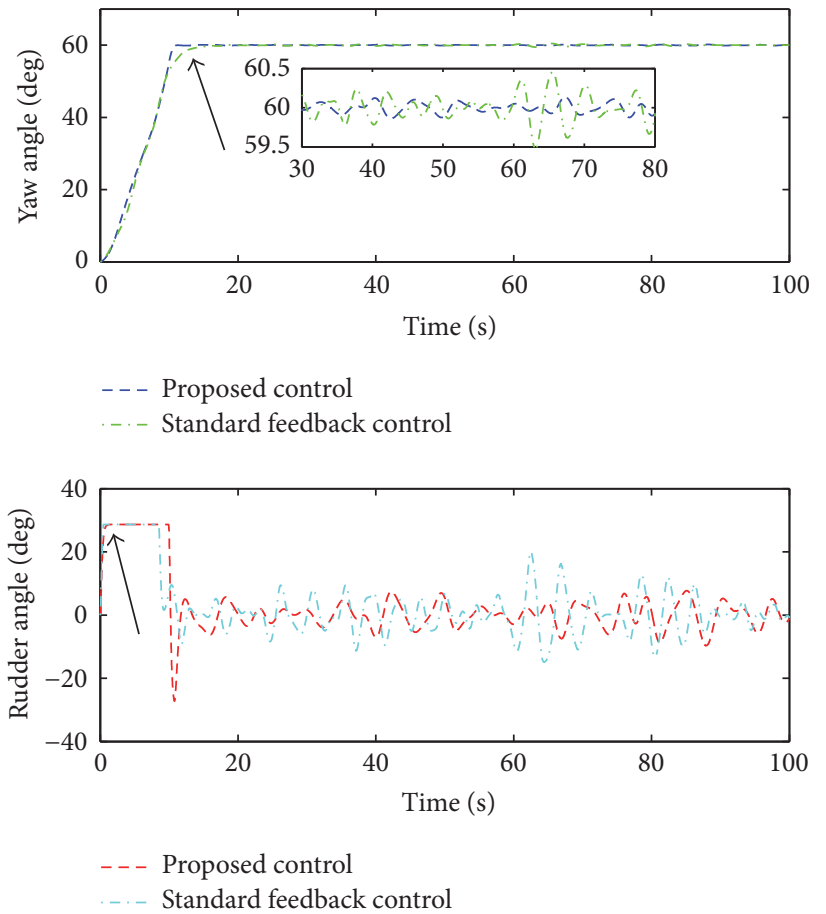

FIGURE 10: Yaw response of different time scale with command course angle of $60^{\circ}$.

the advantages for control design of complicated interconnection systems with different time scale. In future work, a hardware-in-loop simulation testbed will be implemented so that further experiments can be assigned to verify the effectiveness of the proposed methodology. New structures of 

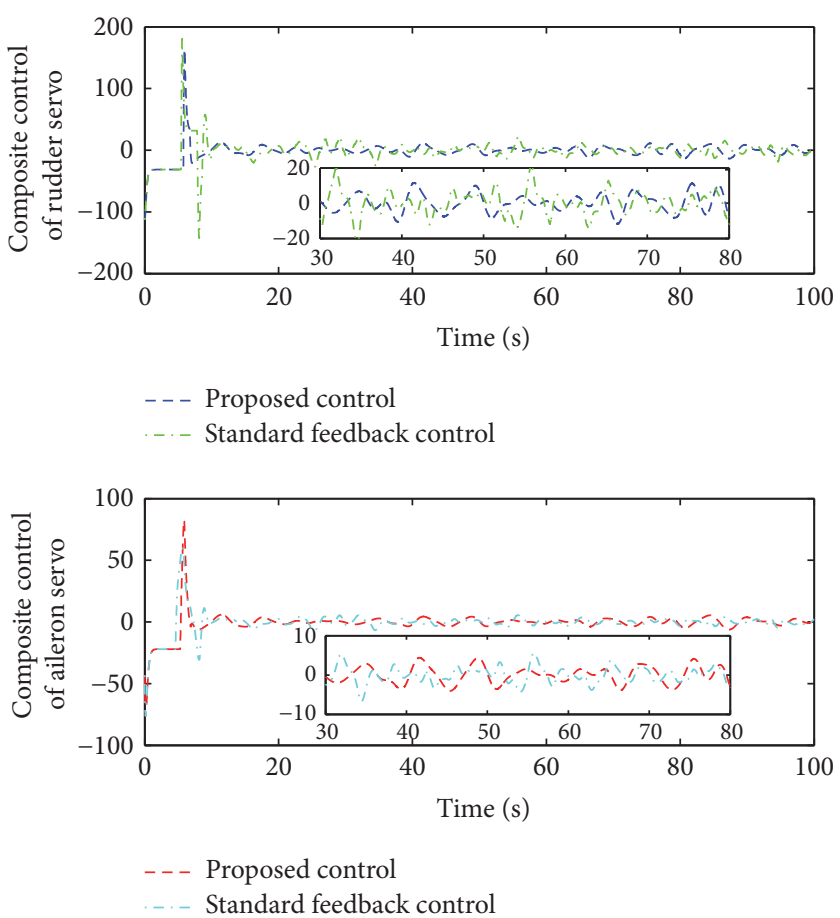

FIGURE 11: Composite control input of the FSHV with command course angle of $-30^{\circ}$.
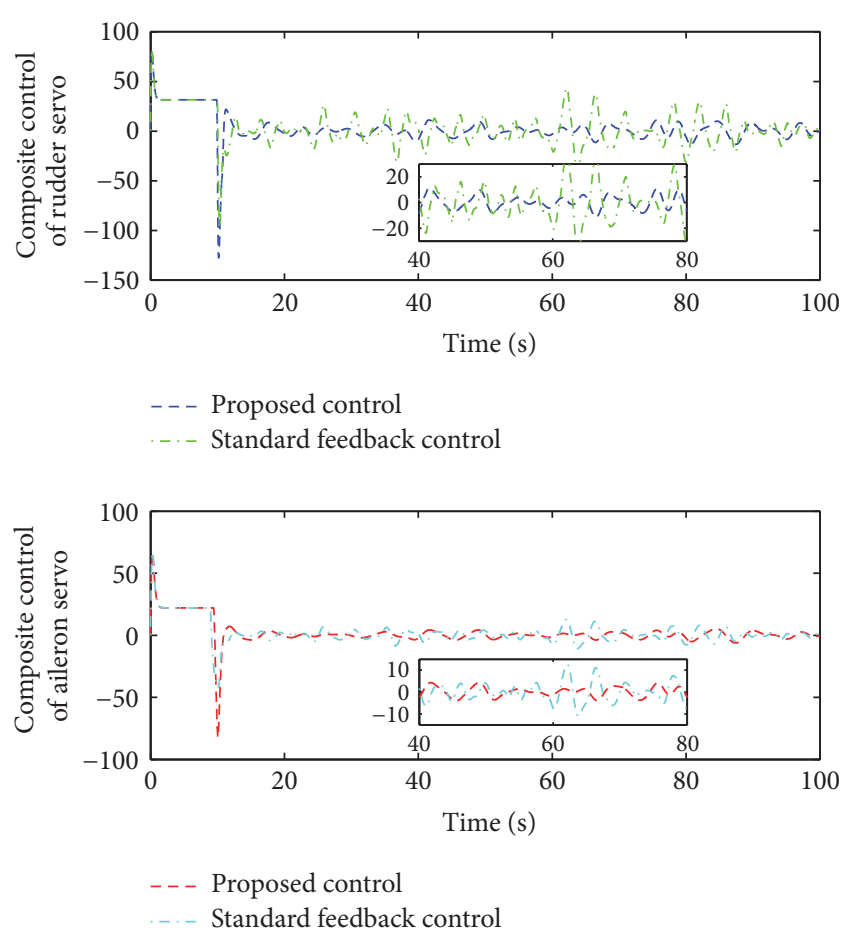

FIGURE 12: Composite control input of the FSHV with command course angle of $60^{\circ}$.

DOB will be discussed to relax the restrictions of the lumped disturbances. And adaptive estimator is to be considered to handle unknown hydrodynamics combined with the RISE feedback.

\section{Conflicts of Interest}

The authors declare that there are no conflicts of interest regarding the publication of this paper.

\section{Acknowledgments}

This work is supported by the National Natural Science Foundation of China under Grant 51579047.

\section{References}

[1] Y. Saito and T. Ikebuchi, "Fully submerged hydrofoil vessel," 1990, 7th Marine Dynamics Symposium on Prediction of Seakeeping Qualities of High-Speed Vessel, pp. 107-141.

[2] H. Imamura, Y. Saito, and M. Asao, "Automatic control system for jetfoil,” Kawasaki Technical Review, pp.107: 1-9, 1990.

[3] S.-H. Kim and H. Yamato, "An experimental study of the longitudinal motion control of a fully submerged hydrofoil model in following seas," Ocean Engineering, vol. 31, no. 5-6, pp. 523-537, 2004.

[4] S.-H. Kim and H. Yamato, "On the design of a longitudinal motion control system of a fully-submerged hydrofoil craft based on the optimal preview servo system," Ocean Engineering, vol. 31, no. 13, pp. 1637-1653, 2004.

[5] S. Liu, C. Xu, and Y. Wang, "Disturbance rejection control for the course keeping of the fully-submerged hydrofoil craft," in Proceedings of the 35th Chinese Control Conference, CCC 2016, pp. 747-751, IEEE, Chengdu, China, July 2016.

[6] T. I. Fossen, Handbook of Marine Craft Hydrodynamics and Motion Control, John Wiley \& Sons, New York, NY, USA, 2011. 
[7] A. Rosales and I. Boiko, "Disturbance attenuation for systems with second-order sliding modes via linear compensators," IET Control Theory \& Applications, vol. 9, no. 4, pp. 526-537, 2015.

[8] J. D. Sánchez-Torres, A. Navarrete-Guzman, G. Rubio-Astorga, and A. G. Loukianov, "High order integral nested sliding mode control," in Proceedings of the 13th International Workshop on Variable Structure Systems, VSS 2014, pp. 1-5, IEEE, Nantes, France, July 2014.

[9] Y. Feng, F. Han, and X. Yu, "Chattering free full-order slidingmode control," Automatica, vol. 50, no. 4, pp. 1310-1314, 2014.

[10] S.-W. Ji, V. P. Bui, B. Balachandran, and Y.-B. Kim, "Robust control allocation design for marine vessel," Ocean Engineering, vol. 63, pp. 105-111, 2013.

[11] K. D. Do and J. Pan, "State- and output-feedback robust pathfollowing controllers for underactuated ships using SerretFrenet frame," Ocean Engineering, vol. 31, no. 5-6, pp. 587-613, 2004.

[12] N. E. Kahveci and P. A. Ioannou, "Adaptive steering control for uncertain ship dynamics and stability analysis," Automatica, vol. 49, no. 3, pp. 685-697, 2013.

[13] P. M. Patre, W. MacKunis, M. Johnson, and W. E. Dixon, "Composite adaptive control for Euler-Lagrange systems with additive disturbances," Automatica, vol. 46, no. 1, pp. 140-147, 2010.

[14] B. Xian, D. M. Dawson, M. S. de Queiroz, and J. Chen, "A continuous asymptotic tracking control strategy for uncertain nonlinear systems," Institute of Electrical and Electronics Engineers. Transactions on Automatic Control, vol. 49, no. 7, pp. 1206-1211, 2004.

[15] W. Ji, A. Wang, and J. Qiu, "Decentralized Fixed-Order Piecewise Affine Dynamic Output Feedback Controller Design for Discrete-Time Nonlinear Large-Scale Systems," IEEE Access, vol. 5, pp. 1977-1989, 2017.

[16] L. J. Zhang, H. M. Jia, and X. Qi, "NNFFC-adaptive output feedback trajectory tracking control for a surface ship at high speed," Ocean Engineering, vol. 38, no. 13, pp. 1430-1438, 2011.

[17] J. Wang, S. Li, J. Yang, B. Wu, and Q. Li, "Extended state observer-based sliding mode control for PWM-based DC-DC buck power converter systems with mismatched disturbances," IET Control Theory and Applications, vol. 9, no. 4, pp. 579-586, 2015.

[18] C. Wang, X. Li, L. Guo, and Y. W. Li, "A nonlinear-disturbanceobserver-based DC-Bus voltage control for a hybrid AC/DC microgrid," IEEE Transactions on Power Electronics, vol. 29, no. 11, pp. 6162-6177, 2014.

[19] H. Sun, S. Li, J. Yang, and L. Guo, "Non-linear disturbance observer-based back-stepping control for airbreathing hypersonic vehicles with mismatched disturbances," IET Control Theory and Applications, vol. 8, no. 17, pp. 1852-1865, 2014.

[20] A. Mohammadi, M. Tavakoli, H. J. Marquez, and F. Hashemzadeh, "Nonlinear disturbance observer design for robotic manipulators," Control Engineering Practice, vol. 21, no. 3, pp. 253-267, 2013.

[21] W.-H. Chen, "Nonlinear disturbance observer-enhanced dynamic inversion control of missiles," Journal of Guidance, Control, and Dynamics, vol. 26, no. 1, pp. 161-166, 2003.

[22] L. Guo and S. Cao, "Anti-disturbance control theory for systems with multiple disturbances: a survey," ISA Transactions, vol. 53, no. 4, pp. 846-849, 2014.

[23] D. Ginoya, P. D. Shendge, and S. B. Phadke, "Sliding mode control for mismatched uncertain systems using an extended disturbance observer," IEEE Transactions on Industrial Electronics, vol. 61, no. 4, pp. 1983-1992, 2014.

[24] X. Lei, Y. Zou, and F. Dong, "A composite control method based on the adaptive RBFNN feedback control and the ESO for twoaxis inertially stabilized platforms," ISA Transactions, vol. 59, pp. 424-433, 2015.

[25] S. Li, J. Yang, W. H. Chen, and X. Chen, Disturbance ObserverBased Control: Methods and Applications, CRC Press, 2014.

[26] T. Li, B. Yu, and B. Hong, "A novel adaptive fuzzy design for path following for underactuated ships with actuator dynamics," in Proceedings of the 2009 4th IEEE Conference on Industrial Electronics and Applications, ICIEA 2009, pp. 2796-2800, chn, May 2009.

[27] H. K. Khalil, Nonlinear Systems Third Edition, Prentice-Hall, Inc, Upper Saddle River, NJ, 2002.

[28] S. Esteban, F. Gordillo, and J. Aracil, “Three-time scale singular perturbation control and stability analysis for an autonomous helicopter on a platform," International Journal of Robust and Nonlinear Control, vol. 23, no. 12, pp. 1360-1392, 2013.

[29] S. Esteban, F. Gavilan, and J. A. Acosta, "Singular perturbation control of the lateral-directional flight dynamics of an UAV," IFAC-PapersOnLine, vol. 28, no. 9, pp. 120-125, 2015.

[30] G. Flores and R. Lozano, "Lyapunov-based controller using singular perturbation theory: An application on a mini-UAV," in Proceedings of the 2013 1st American Control Conference, ACC 2013, pp. 1596-1601, Washington, DC, USA, June 2013.

[31] R.-Y. Ren, Z.-J. Zou, and X.-G. Wang, "A two-time scale control law based on singular perturbations used in rudder roll stabilization of ships," Ocean Engineering, vol. 88, pp. 488-498, 2014.

[32] B. Yi, L. Qiao, and W. Zhang, “Two-time scale path following of underactuated marine surface vessels: Design and stability analysis using singular perturbation methods," Ocean Engineering, vol. 124, pp. 287-297, 2016. 


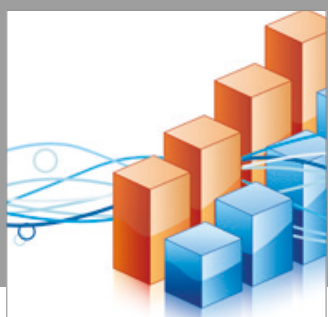

Advances in

Operations Research

vatersals

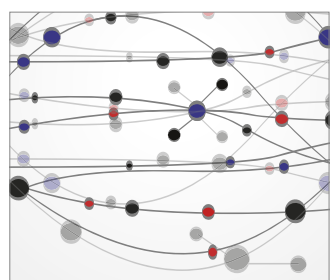

\section{The Scientific} World Journal
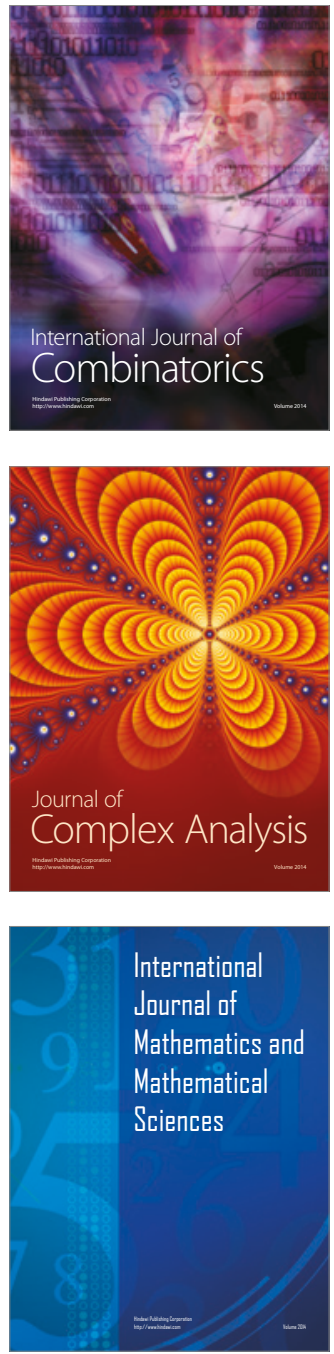
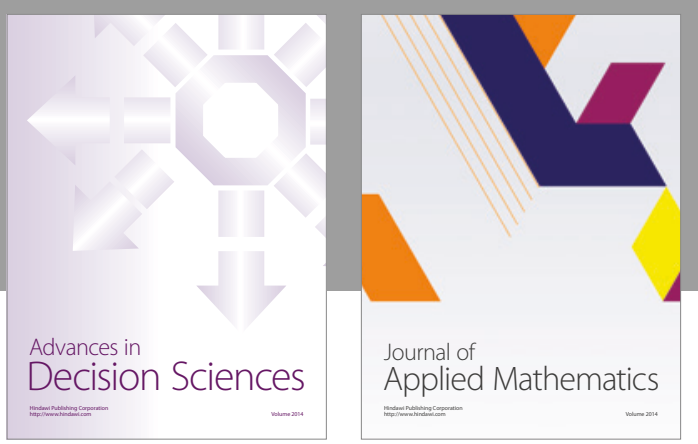

Algebra

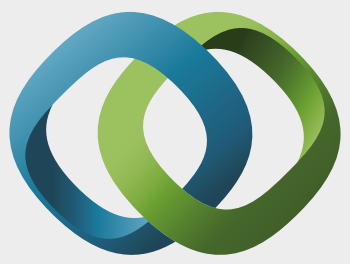

\section{Hindawi}

Submit your manuscripts at

https://www.hindawi.com
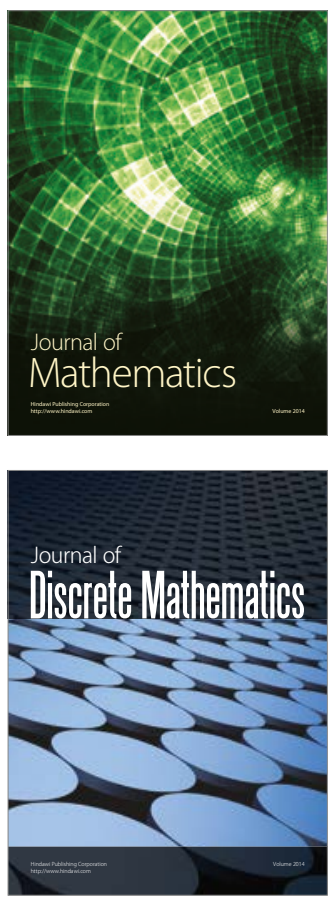

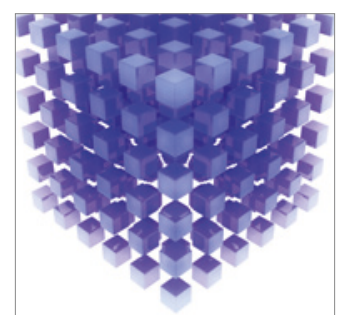

Mathematical Problems in Engineering
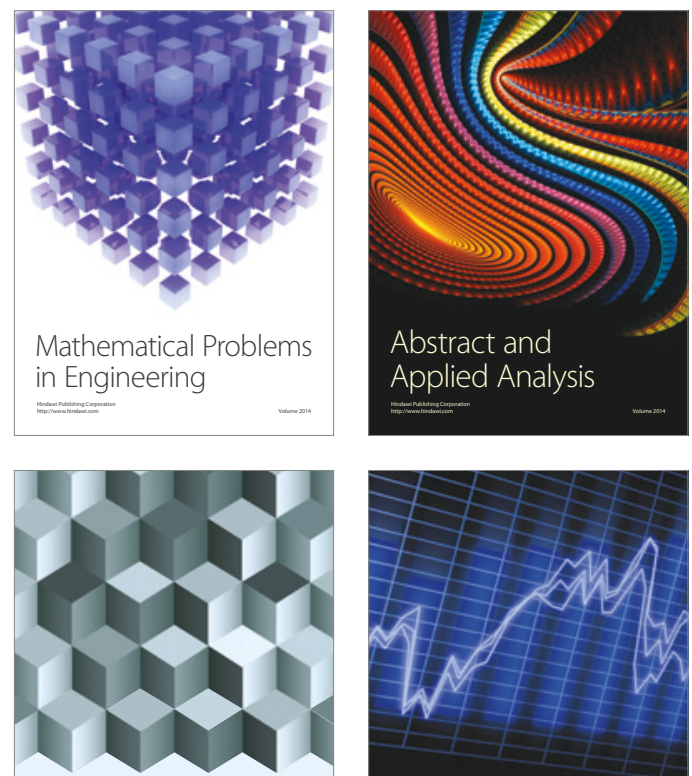

Journal of

Function Spaces

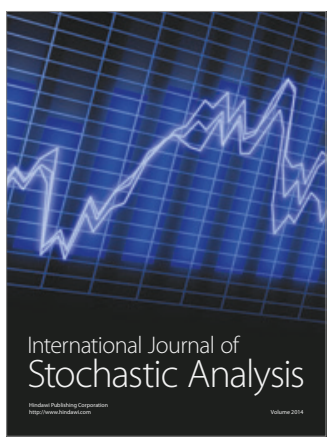

Probability and Statistics
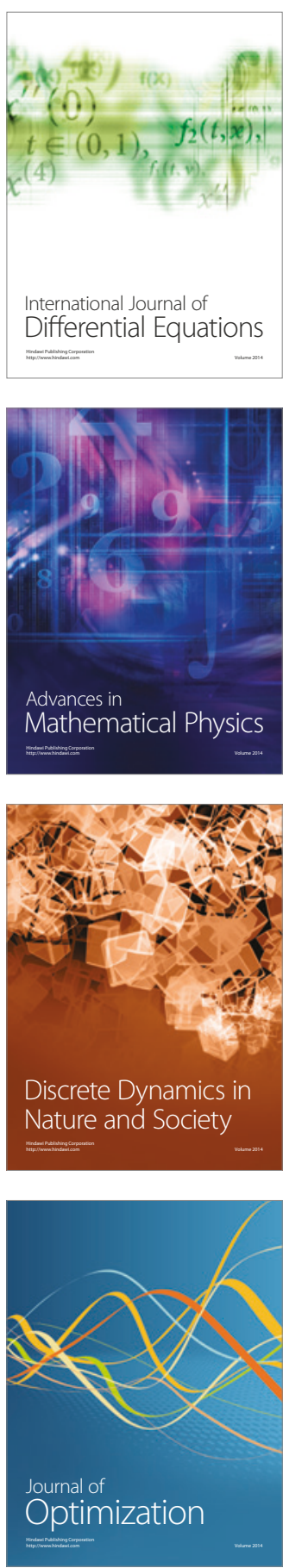